DFPD 95/TH/05

hep-th/9502089

\title{
Algebraic-Geometrical Formulation of Two-Dimensional Quantum Gravity
}

\author{
G. Bonelli, P.A. Marchetti and M. Matone \\ Department of Physics "G. Galilei" - Istituto Nazionale di Fisica Nucleare \\ University of Padova \\ Via Marzolo, 8 - 35131 Padova
}

ITALY

\begin{abstract}
We find a volume form on moduli space of double-punctured Riemann surfaces whose integral satisfies the Painlevé I recursion relations of the genus expansion of the specific heat of 2D gravity. This allows us to express the asymptotic expansion of the specific heat as an integral on an infinite dimensional moduli space in the spirit of Friedan-Shenker approach. We outline a conjectural derivation of such recursion relations using the Duistermaat-Heckman theorem.
\end{abstract}

\footnotetext{
*Partly supported by the European Community Research Programme Gauge Theories, applied supersymmetry and quantum gravity, contract SC1-CT92-0789.

e-mail:

bonelli@ipdgr4.pd.infn.it

marchetti@padova.infn.it

matone@padova.infn.it
} 
1. Important results concerning 2D gravity appeared in the last few years. Investigations of the measure of Liouville quantum gravity were considered in [1] whereas nonperturbative results have been obtained in the framework of matrix models approach [2] and in the topological approach in [3] [4] [5] [6]. The relationships between the different approaches to 2D gravity have been investigated (see [7] for a review), in particular it has been proved [8] that the Liouville action enters in the evaluation of intersection numbers involved in topological gravity. More recently it has been proved that the specific heat of a model of pure gravity can be expressed in terms of a series of integrals on moduli spaces of punctured spheres with the integrand involving the classical Liouville action [9]. In [10 it has been shown that this model corresponds to Liouville quantum gravity with a non trivial $\Theta$-vacuum.

Interesting suggestions on the structure of Liouville path-integral have been considered in [1]. However the explicit form of the measure is still unknown.

Our aim in this paper is to start from the exact results of matrix models and then to write the contribution at genus $h$ to the asymptotic expansion of the specific heat as an integral on $\overline{\mathcal{M}}_{h, 2}$, the compactified moduli spaces of double-punctured Riemann surfaces. Let us shortly outline how this can be achieved. It is well known that the specific heat can be identified with minus the two-puncture correlator $\left\langle\left(\mathcal{O}_{0}\right)^{2}\right\rangle$ and this explains the appearence of $\overline{\mathcal{M}}_{h, 2}$, furthermore it satisfies the Painlevé I (PI) equation. One starts by considering the recursion relations of the asymptotic expansion of PI. In order to reproduce them in terms of integrals on moduli spaces one first notes that the Weil-Petersson two-form $\omega_{W P}$, the natural Kähler form on the moduli space of Riemann surfaces $\Sigma$, has the remarkable property of satisfying the restriction phenomenon. Namely, if we write [11] $\omega_{W P}$ in terms of Fenchel-Nielsen coordinates $l_{j}, \tau_{j}$, the restriction of

$$
\omega_{W P}=\sum_{j} d l_{j} \wedge d \tau_{j}
$$

to the submanifold $l_{k_{1}}=0, \ldots, l_{k_{n}}=0$, is the sum of the Weil-Petersson two-forms on the moduli spaces for the components of the Riemann surface $\Sigma-\left\{\right.$ nodes for $\left.k_{1}, \ldots, k_{n}\right\}$. Second, one notes that the structure of the recursion relations for the asymptotic expansion (see (3)) appears to be related to the structure of the boundary of the moduli space $\overline{\mathcal{M}}_{h, 2}$ involving Riemann surfaces with nodes (see (1)). However, to obtain such recursion relations from the Weil-Petersson two-form using Poincaré duality in the framework of algebraic geometry, one meets the difficulty that the Poincaré dual of $\omega_{W P}$ is not concentrated on the boundary of moduli spaces. This problem can be solved by introducing a volume form $\omega_{W P}^{3 h-2} \wedge \omega_{L}$, 
where the cohomology class $\left[\omega_{L}\right]$ of the two-form $\omega_{L}$ is the Poincaré dual of a divisor $D_{L}$ concentrated at the boundary of $\overline{\mathcal{M}}_{h, 2}$ and this permits the use of the restriction phenomenon property of $\omega_{W P}$. The choice of $D_{L}$ is quite crucial and is determined by the structure of the theory. This algebraic-geometrical formulation derived directly from the matrix model strengths the connection between topological and Liouville gravity.

Recovering the volume form on $\overline{\mathcal{M}}_{h, 2}$ associated to the PI, allows us to write the asymptotic series as a unique integral of a suitable volume form on an infinite dimensional space of double-punctured Riemann surfaces, in the spirit of the Friedan-Shenker program for 2D quantum gravity. This integral is the asymptotic counterpart of an analogous integral on an infinite dimensional space of all punctured Riemann spheres considered in 10 representing the specific heat in the strong coupling region.

2. Let us shortly recall some basic facts about the moduli space of stable curves $\overline{\mathcal{M}}_{h}$, the Deligne-Knudsen-Mumford compactification of moduli space, and the specific heat of 2D gravity.

$\overline{\mathcal{M}}_{h}$ is a projective variety and its boundary $\partial \overline{\mathcal{M}}_{h}=\overline{\mathcal{M}}_{h} \backslash \mathcal{M}_{h}$, called the compactification divisor, decomposes into a union of divisors $D_{0}, \ldots, D_{[h / 2]}$ which are subvarieties of complex codimension one. A Riemann surface $\Sigma$ belongs to $D_{k} \cong \overline{\mathcal{M}}_{h-k, 1} \times \overline{\mathcal{M}}_{k, 1}, k>0$ if it has one node separating it into two components of genus $k$ and $h-k$. The locus in $D_{0} \cong \overline{\mathcal{M}}_{h-1,2}$ consists of surfaces that become, on removal of the node, genus $h-1$ double punctured surfaces. Surfaces with multiple nodes lie in the intersections of the $D_{k}$ 's. The compactified moduli space $\overline{\mathcal{M}}_{h, n}$ of Riemann surfaces with $n$-punctures $z_{1}, \ldots, z_{n}$ is defined analogously to $\overline{\mathcal{M}}_{h}$. In particular for $n=2$ we have

$$
\partial \overline{\mathcal{M}}_{h+1,2}=e_{0}^{(h+1)} \overline{\mathcal{M}}_{h, 4}+\sum_{k=1}^{h} e_{k}^{(h+1)} \overline{\mathcal{M}}_{h-k+1,2} \times \overline{\mathcal{M}}_{k, 2}+\sum_{k=0}^{h} o_{k}^{(h+1)} \overline{\mathcal{M}}_{h-k+1,1} \times \overline{\mathcal{M}}_{k, 3},
$$

in the sense of cycles on orbifolds, where the coefficients are combinatorial factors.

The specific heat of pure gravity satisfies the PI, $\mathcal{Z}^{2}(t)-\mathcal{Z}^{\prime \prime}(t) / 3=t$, where $t \equiv \lambda_{1}^{R} g_{s}^{-4 / 5}$, with $\lambda_{1}^{R}$ the renormalized cosmological constant and $g_{s}$ the string coupling constant. The asymptotic (genus) expansion of $\mathcal{Z}(t)$ has the form

$$
\mathcal{Z}(t) \sim \sum_{h=0}^{\infty} e^{2 i \Theta(1-h)} Z_{h} t^{-5 h / 2+1 / 2}, \quad t \rightarrow+\infty
$$

with the coefficients $Z_{h}$ satisfying the recursion relations

$$
Z_{0}=1, \quad Z_{h+1}=\frac{25 h^{2}-1}{24} Z_{h}-\frac{1}{2} \sum_{k=1}^{h} Z_{h-k+1} Z_{k}, \quad \Theta=0, \pi / 2,
$$


where $\Theta=0$ corresponds to ordinary 2D gravity [2], whereas $\Theta=\pi / 2$ corresponds to the model with a non trivial $\Theta$-vacuum proposed in [10].

In terms of the free energy $F$, the $n$-puncture correlator is given by [2]

$$
\left\langle\left(\mathcal{O}_{0}\right)^{n}\right\rangle=-\frac{d^{n} F(t)}{d t^{n}}
$$

and $\mathcal{Z}(t)=F^{\prime \prime}(t)$.

For future purpose we write down the genus expansion of the 4-puncture correlator

$$
\left\langle\left(\mathcal{O}_{0}\right)^{4}\right\rangle=\frac{d^{2}}{d t^{2}}\left\langle\left(\mathcal{O}_{0}\right)^{2}\right\rangle \sim-\sum_{h=0}^{\infty} e^{2 i \Theta(1-h)} W_{h} t^{-5 h / 2-3 / 2}, \quad t \rightarrow+\infty, \quad W_{h}=\frac{25 h^{2}-1}{4} Z_{h} .
$$

3. We now find a representation of $Z_{h}$ in the form $\int_{\overline{\mathcal{M}}_{h, 2}} \omega^{(h)}$, with $\omega^{(h)}$ a $(6 h-2)$-form on $\overline{\mathcal{M}}_{h, 2}$. We assume $\omega^{(h)} \equiv\left(\omega_{W P}^{(h, 2)}\right)^{3 h-2} \wedge \omega_{L}^{(h)} /(3 h-1)$ ! with $\omega_{L}^{(h)}$ a two-form whose explicit expression will be given later and $\omega_{W P}^{(h, n)}$ the Weil-Petersson two-form on $\overline{\mathcal{M}}_{h, n}$ divided by $\pi^{2}$. We set

$$
Z_{h}=\frac{1}{(3 h-1) !} \int_{\overline{\mathcal{M}}_{h, 2}}\left(\omega_{W P}^{(h, 2)}\right)^{3 h-2} \wedge \omega_{L}^{(h)}, \quad h>0
$$

with initial condition $Z_{0}=1$.

We now define a divisor $D_{L}^{(h)}$, which we call "Liouville divisor", as the $(6 h-4)$-cycle

$$
D_{L}^{(h)}=c_{0}^{(h)} \overline{\mathcal{M}}_{h-1,4}+\sum_{k=1}^{h-1} c_{k}^{(h)} \overline{\mathcal{M}}_{h-k, 2} \times \overline{\mathcal{M}}_{k, 2}
$$

where the coefficients $c_{k}^{(h)}$ will be given later. We identify $\left[\omega_{L}^{(h)}\right]$ as the Poincaré dual to $D_{L}^{(h)}$, i.e. $\left[\omega_{L}^{(h)}\right]=c_{1}\left(\left[D_{L}^{(h)}\right]\right)$ where, as usual, $[D]$ denotes the line bundle associated to a given divisor $D$ (see for example [12]) and $c_{1}$ denotes the first Chern class.

We now fix the $c_{k}^{(h)}$ 's by requiring that $Z_{h}$ 's defined in (6) satisfy the recursion relations (3). Two facts are crucial to obtain recursion relations: first, in evaluating the relevant integrals will appear only the components of the boundary $\partial \overline{\mathcal{M}}_{h, 2}$ of the form $\overline{\mathcal{M}}_{h-k, i} \times \overline{\mathcal{M}}_{k, j}$ with $i=j=2$ and $\overline{\mathcal{M}}_{h-1,4}$, second, $\omega_{W P}^{(h, 2)}$ satisfies the restriction phenomenon mentioned above. In particular, considering the natural embedding

$$
i: \overline{\mathcal{M}}_{k, 2} \rightarrow \overline{\mathcal{M}}_{k, 2} \times * \rightarrow \overline{\mathcal{M}}_{k, 2} \times \overline{\mathcal{M}}_{h-k, 2} \rightarrow \partial \overline{\mathcal{M}}_{h, 2} \rightarrow \overline{\mathcal{M}}_{h, 2}, \quad h>k
$$

one has by [1] [13]

$$
\left[\omega_{W P}^{(k, 2)}\right]=i^{*}\left[\omega_{W P}^{(h, 2)}\right]
$$


and similarly for $\left[\omega_{W P}^{(h-1,4)}\right]$. By Poincaré duality one obtains

$$
\int_{\overline{\mathcal{M}}_{h, 2}}\left(\omega_{W P}^{(h, 2)}\right)^{3 h-2} \wedge \omega_{L}^{(h)}=\left[\omega_{W P}^{(h, 2)}\right]^{3 h-2} \cap\left[D_{L}^{(h)}\right]
$$

so that by (8)-(9) it follows that

$$
\begin{gathered}
Z_{h+1}=\frac{1}{(3 h+2) !}\left\{c_{0}^{(h+1)}\left[\omega_{W P}^{(h, 4)}\right]^{3 h+1} \cap\left[\overline{\mathcal{M}}_{h, 4}\right]+\right. \\
\left.\sum_{k=1}^{h} c_{k}^{(h+1)}\left[\omega_{W P}^{(h-k+1,2)}+\omega_{W P}^{(k, 2)}\right]^{3 h+1} \cap\left[\overline{\mathcal{M}}_{h-k+1,2} \times \overline{\mathcal{M}}_{k, 2}\right]\right\} .
\end{gathered}
$$

The only contribution in the second term in the RHS comes from the $\left(\omega_{W P}^{(h-k+1,2)}\right)^{3(h-k)+2} \wedge$ $\left(\omega_{W P}^{(k, 2)}\right)^{3 k-1}$ term, therefore we have

$$
\begin{gathered}
Z_{h+1}=\frac{1}{(3 h+2) !}\left\{c_{0}^{(h+1)} \int_{\overline{\mathcal{M}}_{h, 4}}\left(\omega_{W P}^{(h, 4)}\right)^{3 h+1}+\right. \\
\left.\sum_{k=1}^{h} c_{k}^{(h+1)}\left(\begin{array}{l}
3 h+1 \\
3 k-1
\end{array}\right) \int_{\overline{\mathcal{M}}_{h-k+1,2}}\left(\omega_{W P}^{(h-k+1,2)}\right)^{3(h-k)+2} \int_{\overline{\mathcal{M}}_{k, 2}}\left(\omega_{W P}^{(k, 2)}\right)^{3 k-1}\right\} .
\end{gathered}
$$

The recursion relations (12) coincide with (3) if we set

$c_{0}^{(h+1)}=\frac{(3 h+2)}{6} \frac{W_{h}}{a_{h, 4}}=\frac{(3 h+2)}{6} \frac{25 h^{2}-1}{4 a_{n, 4}} Z_{h}, \quad c_{k}^{(h+1)}=-\frac{3 h+2}{2}\left(\frac{Z_{k} Z_{h-k+1}}{a_{k, 2} a_{h-k+1,2}}\right), \quad k>0$,

where

$$
a_{k, n}=\frac{1}{(3 k-3+n) !} \int_{\overline{\mathcal{M}}_{k, n}}\left(\omega_{W P}^{(k, n)}\right)^{3 k-3+n},
$$

is the Weil-Petersson volume of $\overline{\mathcal{M}}_{k, n}$ times $\left(1 / \pi^{2}\right)^{3 k-3+n}$ and $W_{h}$ has been defined in (5). Notice that all the coefficients $c_{k}^{(h)}$ are rational numbers so that $D_{L}$ defines a rational homology class and the above computations can be interpreted in the sense of rational intersection theory.

Comparing with (2) with (6) we have asymptotically

$$
\mathcal{Z}(t) \sim e^{2 i \Theta} t^{\frac{1}{2}}+\sum_{h=1}^{\infty} \frac{e^{2 i \Theta(1-h)} t^{-\frac{5}{2} h+\frac{1}{2}}}{(3 h-1) !} \int_{\overline{\mathcal{M}}_{h, 2}}\left(\omega_{W P}^{(h, 2)}\right)^{3 h-2} \wedge \omega_{L}^{(h)}
$$

If one is able to express the coefficients $Z_{h}$ in terms of the expansion around $t=0$ [9] [10] then, since the divisor of the expansions around $t=0$ and $t \rightarrow+\infty$ are known, it should be also possible to understand in more detail the reduction mechanism from genus $h$ to 
punctured spheres. We remark that the structure used in the derivation of the recursion relations can be generalized to integrals of $\overline{\mathcal{M}}_{h, n}$ yielding recursion relations for $n$-puncture correlator. Furthermore, together with a suitable choice of the divisors at infinity, the use of the restriction phenomenon seems to be useful to investigate intersection theory on $\overline{\mathcal{M}}_{h, n}$.

4. As in [10 one can construct an infinite dimensional moduli space of Riemann surfaces including those with infinitely many handles via the following inductive limit. We consider the embedding

$$
i_{h}: \overline{\mathcal{M}}_{h, 2} \rightarrow \overline{\mathcal{M}}_{h+1,2}, \quad h>0,
$$

and for $q \in \mathbf{R}_{+}$we define

$$
\overline{\mathcal{M}}_{\infty, 2}(q)=\coprod_{h=1}^{\infty}\left(\overline{\mathcal{M}}_{h, 2} \times\left[0, q^{h}\right]\right) /\left(\overline{\mathcal{M}}_{h, 2}, q^{h}\right) \sim\left(i_{h}\left(\overline{\mathcal{M}}_{h, 2}\right), 0\right)
$$

Let $d y$ denote the Lebesgue measure on $\mathbf{R}$ and define the indefinite rank form

$$
\mathcal{T}_{\infty}=\sum_{h=1}^{\infty} \frac{e^{2 i \Theta(1-h)} \omega_{W P}^{(h, 2)}{ }^{3 h-2} \wedge \omega_{L}^{(h)}}{(3 h-1) !} \wedge d y
$$

then asymptotically

$$
\mathcal{Z}(t) \sim e^{2 i \Theta} t^{1 / 2}+t^{1 / 2} \int_{\overline{\mathcal{M}}_{\infty, 2}\left(t^{-5 / 2}\right)} \mathcal{T}_{\infty}
$$

One can give a meaning to the RHS of (17) in terms of a perturbation series. If the asymptotic series is Borel summable, as presumably happens in 2D gravity with $\Theta$-vacua, a more precise meaning can be given via Borel summation.

Equation (17), expresses the asymptotic behaviour of the specific heat of nonperturbative 2D quantum gravity as an integral on an infinite dimensional space involving moduli spaces of all double-punctured Riemann surfaces, and hence can be interpreted in a sense as a kind of realization of the Friedan-Shenker program [14] in the asymptotic region.

5. We now give a conjectural argument that could relate our Ansatz (6) to the pathintegral approach to Liouville gravity. In this line of thought a key step is the DuistermaatHeckman (DH) [15] which roughly speaking corresponds to the following statement. Let $X$ be a $2 n$-dimensional symplectic manifold with symplectic form $\omega$ and $H$ a Hamiltonian on $X$. Then integrals such as

$$
\frac{1}{n !} \int_{X} \omega^{n} e^{-H}
$$


only depend on the behaviour of the integrand near the critical points of the flow of the Hamiltonian vector field. The point is that in a path-integral approach one expects that the contribution at genus $h$ to the two-puncture correlation function of $2 \mathrm{D}$ gravity is given by

$$
\left\langle\left(\mathcal{O}_{0}\right)^{2}\right\rangle_{h}=\frac{1}{(3 h-1) !} \int_{\overline{\mathcal{M}}_{h, 2}}\left(\omega_{W P}^{(h, 2)}\right)^{3 h-1} e^{-H}
$$

where $H$ is an "effective action" arising from the integration in the path-integral at fixed moduli in $\overline{\mathcal{M}}_{h, 2}$. The two-form $\omega_{W P}^{(h, 2)}$ is symplectic on $\overline{\mathcal{M}}_{h, 2}$, regular in the interior and extending as a current to the boundary, therefore, regarded as a map from $T^{*} \overline{\mathcal{M}}_{h, 2}$ to $T \overline{\mathcal{M}}_{h, 2},\left(\omega_{W P}^{(h, 2)}\right)^{-1}$ has zeroes only on $\partial \overline{\mathcal{M}}_{h, 2}$. Furthermore, since $\omega_{W P}^{(h, 2)}$ is a Kähler form, the Hamiltonian vector field is given by $\left(\omega_{W P}^{(h, 2)}\right)^{-1} d H$ so that the flow of the Hamiltonian vector field has critical points at $\partial \overline{\mathcal{M}}_{h, 2}$. Let us assume that DH applies to the integral (18) and furthermore it gets contribution only from the critical points in the component of $\partial \overline{\mathcal{M}}_{h, 2}$ whose factor contain an even number of punctures. Then one expects

$$
\begin{gathered}
\left\langle\left(\mathcal{O}_{0}\right)^{2}\right\rangle_{h+1} \sim \alpha_{h+1} \frac{1}{(3 h+1) !} \int_{\overline{\mathcal{M}}_{h, 4}}\left(\omega_{W P}^{(h, 4)}\right)^{3 h+1} e^{-H}+ \\
\beta_{h+1} \sum_{k=1}^{h}\left[\frac{1}{[3(h-k)+2] !} \int_{\overline{\mathcal{M}}_{h-k+1,2}}\left(\omega_{W P}^{(h-k+1,2)}\right)^{3(h-k)+2} e^{-H}\right] . \\
{\left[\frac{1}{(3 k-1) !} \int_{\overline{\mathcal{M}}_{k, 2}}\left(\omega_{W P}^{(k, 2)}\right)^{3 k-1} e^{-H}\right]=\alpha_{h+1}\left\langle\left(\mathcal{O}_{0}\right)^{4}\right\rangle_{h}+\beta_{h+1} \sum_{k=1}^{h}\left\langle\left(\mathcal{O}_{0}\right)^{2}\right\rangle_{h-k+1}\left\langle\left(\mathcal{O}_{0}\right)^{2}\right\rangle_{k},}
\end{gathered}
$$

where $\alpha_{h+1}, \beta_{h+1}$ are possibly $t$-dependent coefficients. One can remark the analogy with the recursion relations derived from $\mathrm{KdV}$ in topological gravity. Let us introduce the cohomology classes $\left[\eta^{(h, k)}\right] \in H^{2}\left(\overline{\mathcal{M}}_{h, 2}\right), k=0, \ldots, h$, Poincaré dual of $\overline{\mathcal{M}}_{h-1,4}, \overline{\mathcal{M}}_{h-k, 2} \times \overline{\mathcal{M}}_{k, 2}, k=$ $1, \ldots, h$. Introducing the normalized Weil-Petersson volumes $a_{k, n}$ and using (5), one obtains for the asymptotic behaviour of the correlations

$$
\begin{gathered}
\left\langle\left(\mathcal{O}_{0}\right)^{2}\right\rangle_{h+1} \sim \frac{1}{(3 h+2) !} \int_{\overline{\mathcal{M}}_{h+1,2}}\left(\omega_{W P}^{(h+1,2)}\right)^{3 h+1} \wedge\left[\alpha_{h+1} \frac{25 h^{2}-1}{4} \frac{\left\langle\left(\mathcal{O}_{0}\right)^{2}\right\rangle_{h}}{a_{h, 4}} \eta^{(h+1,0)}+\right. \\
\left.\beta_{h+1} \sum_{k=1}^{h} \frac{\left\langle\left(\mathcal{O}_{0}\right)^{2}\right\rangle_{h-k+1}}{a_{h-k+1,2}} \frac{\left\langle\left(\mathcal{O}_{0}\right)^{2}\right\rangle_{k}}{a_{k, 2}} \eta^{(h+1, k)}\right] .
\end{gathered}
$$

Since the asymptotic expression of $\left\langle\left(\mathcal{O}_{0}\right)^{2}\right\rangle_{h}$ evaluated at $t=1$ is equal to $-Z_{h}$, by setting $t=1$ in (20) we derive

$$
Z_{h} \sim \frac{1}{(3 h-1) !} \int_{\overline{\mathcal{M}}_{h, 2}}\left(\omega_{W P}^{(h, 2)}\right)^{3 h-2} \wedge \omega^{(h)}
$$


with $\omega^{(h)}$ a two-form given by

$$
\omega^{(h+1)}=\alpha_{h+1} \frac{25 h^{2}-1}{4} \frac{Z_{h}}{a_{h, 4}} \eta^{(h+1,0)}-\beta_{h+1} \sum_{k=1}^{h} \frac{Z_{h-k+1}}{a_{h-k+1,2}} \frac{Z_{k}}{a_{k, 2}} \eta^{(h+1, k)},
$$

exhibiting the same structure of the two-form $\omega_{L}^{(h+1)}$ introduced in the Ansatz (6) with the coefficients $c_{k}^{(h+1)}$ given by (13).

6. The above results can be seen as a first step to recover the full structure of Liouville theory. To see this note that divisors at infinity of moduli spaces are related to determinant line bundles $\lambda_{k}$ and Weil-Petersson two-form $\omega_{W P}$. For example in $\overline{\mathcal{M}}_{h}$ one has for $h>2$ 11

$$
c_{1}\left(\lambda_{H}\right)=\frac{1}{24}\left[\omega_{W P} / \pi^{2}\right]+\frac{1}{12} D_{0}+\frac{1}{24} D_{1}+\frac{1}{12} D_{2}+\cdots+\frac{1}{12} D_{[h / 2]},
$$

where $\lambda_{H} \equiv \lambda_{1}$ is the Hodge bundle. The structure (23) together with the Mumford isomorphism $\lambda_{k} \cong \lambda_{1}^{6 k^{2}-6 k+1}$ are basic in the description of the Polyakov volume form $d \pi_{h}$ of the critical bosonic string [16] 17]

$$
Z=\int_{\overline{\mathcal{M}}_{h}} d \pi_{h}
$$

where $d \pi_{h}=e^{\beta(2-2 h)}|\mu|^{2}$, with $\mu$ the Mumford form. The Weil-Petersson volume form is associated to the ghost zero modes whereas sections of determinant line bundles $\lambda_{k}$ arise from path integral on $b$ - $c$ systems of weight $k$. In the description of the algebraic-geometrical structure of the specific heat we recovered the divisors at infinity and the Weil-Petersson two-form. Generalizing the structure (23) to the case of $\overline{\mathcal{M}}_{h, n}$ one could expect to be able to describe the cohomology structure of $Z_{h}$ directly in terms of Weil-Petersson two-form and determinant line bundles, and then to recover the structure of the Liouville path-integral, problem pioneered in [四], associated to matrix models. In other words this suggests that it could be possible to reconstruct the full structure of the theory starting from nonperturbative results and then going back to the continuum formulation. 


\section{References}

[1] F. David, Mod. Phys. Lett. A3 (1988) 1651.

J. Distler and H. Kawai, Nucl. Phys. B321 (1989) 509.

[2] E. Brézin and V. Kazakov, Phys. Lett. 236B (1990) 144.

M. Douglas and S. Shenker, Nucl. Phys. B335 (1990) 635.

D. Gross and A. Migdal, Phys. Rev. Lett. 64 (1990) 127; Nucl. Phys. 340 (1990) 333.

[3] E. Witten, Nucl. Phys. B340 (1990) 281; Surv. Diff. Geom. 1 (1991) 243.

R. Dijkgraaf and E. Witten, Nucl. Phys. B342 (1990) 486.

[4] E. Verlinde and H. Verlinde, Nucl. Phys. B348 (1991) 457.

[5] M. Kontsevich, Funk. Anal. Prilozh. 25 (1991) 50; Comm. Math. Phys. 147 (1992) 1.

[6] C. Itzykson and J.-B. Zuber, Int. J. Mod. Phys. A7 (1992) 5661. P. Di Francesco, C. Itzykson and J.-B. Zuber, Comm. Math. Phys. 151 (1993) 193. P. Di Francesco, Observables in The Kontsevich model, to appear in Low dimensional topology and gravity, Cambridge University Press (1993).

[7] L. Alvarez-Gaumé, Random Surfaces, Statistical Mechanics And String Theory, Lausanne lectures, winter 1990. P. Ginsparg, Matrix Models Of $2 D$ Gravity, Trieste Lectures, LA-UR-91-4101, hepth/9112013. E. Martinec, An Introduction To 2D Gravity And Solvable String Models, Trieste Lectures, RU-91-51, hepth/9112019. A. Morozov, Integrability And Matrix Models, ITEP-M2/93, hepth/9303139. P. Ginsparg and G. Moore, Lectures On 2D Gravity And 2D String Theory, hepth/9304011, Tasi Lectures 1992, ed. J. Harvey and J. Polchinski, World Scientific. P. Di Francesco, P. Ginsparg and J. Zinn-Justin, 2D Gravity And Random Matrices, LA-UR-93-1722, SPhT/93-061, hepth/9306153.

[8] M. Matone, Int. J. Mod. Phys. A10 (1995) 289.

[9] M. Matone, Nonperturbative Model of Liouville Gravity, hepth/9402081.

[10] G. Bonelli, P.A. Marchetti and M. Matone, Phys. Lett. 339B (1994) 49.

[11] S.A. Wolpert, Ann. Math. 118 (1983) 491. 
[12] Ph. Griffiths and J. Harris, "Principles of Algebraic Geometry", John Wiley and Sons, 1978.

[13] S.A. Wolpert, Amer. J. Math. 107 (1985) 969.

[14] D. Friedan and S.H. Shenker, Phys. Lett. 175B (1986) 287; Nucl. Phys. B281 (1987) 509.

[15] J.J. Duistermaat and G.J. Heckman, Invent. Math. 69 (1982) 259.

[16] A.A. Belavin and V.A. Knizhnik, Phys. Lett. 168B (1986) 201.

[17] A.A. Beilinson and Yu. I. Manin, Comm. Math. Phys. 107 (1986) 359. 